\title{
Influence of Partial Cross-Linking Degree on Basic Physical Properties of RTM6 Epoxy Resin
}

\author{
Judith Moosburger-Will, Michael Greisel, Markus G. R. Sause, Robert Horny, Siegfried Horn \\ Experimental Physics II, Institute of Physics, University of Augsburg, 86135 Augsburg, Germany \\ Correspondence to: J. Moosburger-Will (E-mail: judith.will@physik.uni-augsburg.de)
}

\begin{abstract}
Thermo-physical and mechanical properties of partially and completely cross-linked RTM6 epoxy resin samples in the glassy state have been investigated. A significant dependence of glass transition temperature, density, and modulus on the curing history and the curing degree is found. Density and modulus decrease with increasing curing degree and show a step-like irregularity in the so called transition region, which is related to the transition from rubber to glassy state during cross-linking and the starting of structural relaxation processes. The relationship between the thermo-physical and mechanical properties, which is important for the development of new processing routes for fiber reinforced polymers, is addressed.
\end{abstract}

\section{INTRODUCTION}

Tetra-functional epoxy resins are widely used in aerospace industry for production of advanced composite materials, primarily carbon fiber reinforced polymers (CFRP). Also in the fields of transportation and construction a growing market with numerous technical applications exists. ${ }^{1,2}$ After infiltration of the dry fiber fabric with the matrix polymer, the thermoset is cross-linked in a thermal curing process. The reactivity of the fully cross-linked epoxy resin is very low. Partial cross-linking by modified curing cycles maintains a particular rest reactivity of the resin and opens new options for CFRP production. For example the joining of partially cured and fresh resin systems can be realized. This allows an increased level of integral construction of CFRP components, which is a highly relevant aspect in aerospace industry. ${ }^{3}$ A subsequent final curing cycle guarantees the complete cross-linking of the new component.

The physical properties of a thermosetting resin in the cured state depend on several factors, e.g. the chemical structure of the polymer, the type and concentration of the hardener, and the thermal cycle used for curing. For a given chemical composition in particular the curing parameters, namely the curing temperatures, heating rates, and curing durations are important. During the curing process a chemical reaction between epoxy groups and reactive groups of the hardener takes place. Depending on the curing treatment the material will pass either from liquid through rubber to glassy state, or from liquid to rubber state or from liquid to glassy state. ${ }^{4}$ Annealing of a glassy poly- mer at temperatures below its glass transition temperature results in physical ageing processes. ${ }^{5-7}$ Such different curing and annealing cycles will result in different materials properties of the cured thermoset. ${ }^{8}$ Thus, to consider variations of existing curing cycles, it is essential to know the relation between basic thermo-physical and mechanical properties, the curing history and the cross-linking degree. In particular the interdependence of glass transition temperature, curing degree, density, elastic modulus, and water absorption of partially and fully cured epoxy resins is of interest and subject to actual research activities. $^{8-13}$ However, because of the large variety of combinations of resin, hardener and curing cycles, a general systematic assessment of such interdependences is difficult.

In epoxy resins with functionality higher than two, a threedimensional network forms during curing. For such epoxy resins a decrease of density and modulus with increasing crosslinking of the polymer chains is observed in the glassy state (above the gelation point), caused by the reduced possibility of molecular rearrangements. ${ }^{10,14}$ Also di-functional epoxy resins, cured with hardeners of functionality higher than two form three-dimensional networks. Here, a decrease of density and modulus as a function of curing degree is also reported for some systems, ${ }^{9-12,15}$ in one case with a step-like irregularity. ${ }^{6}$ For other di-functional network polymers an increase in density and modulus is observed. ${ }^{14}$ Linear systems, with a functionality of epoxy and hardener of two, are characterized by an increase of density with curing degree. ${ }^{10}$ Generally, relaxation processes 


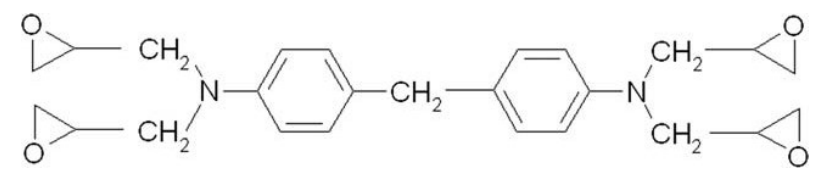

Figure 1. Chemical structure of epoxy resin tetraglycidyl methylene dianiline.

because of physical ageing of the cured epoxy resin result in density and modulus increases. ${ }^{5-7}$ Also, a low density of the cured polymer is correlated to high water absorption. ${ }^{12,13}$

In this work, the material properties of partially cross-linked tetra-functional epoxy resin HexFlow (B) RTM6 samples are investigated, which are produced by isothermal curing cycles at reduced temperatures. Isothermal curing was used to obtain comparable chemical reaction processes for all curing degrees. ${ }^{12}$ Depending on the curing degree the chosen curing treatment involves a liquid-rubber or a liquid-rubber-glass transition during curing. The kind of transition has a high impact on the physical properties of the resulting partially cross-linked epoxy resin samples in their glassy state and promises high potential for different fields of applications. Thermo-physical and mechanical properties of partially and completely cross-linked RTM6 resin samples were investigated as a function of curing degree focusing on glass transition temperature, density and elastic modulus. To determine the elastic modulus the micromechanical method of nanoindentation is used and is compared to results of macroscopic mechanical tests.

\section{EXPERIMENTAL}

\section{Production of Partially and Fully Cross-Linked RTM6 Epoxy Resin Samples}

All resin samples analyzed in this work are manufactured by the mono-component epoxy resin system HexFlow ( ${ }^{\circ}$ RTM6 distributed by the Hexcel Corporation. ${ }^{16}$ It is composed of the tetra-functional epoxy resin tetraglycidyl methylene dianiline (see Figure 1) and the hardeners 4,4'-methylenebis(2,6-diethylaniline) and 4,4'-methylenebis(2-isopropyl-6-methylaniline) (see Figure 2).

Modified curing cycles are used to obtain resin samples with defined curing degrees. The heating and cooling rates always were set to $2^{\circ} \mathrm{C} / \mathrm{min}$. All cycles start with an isothermal curing period at a temperature of $120^{\circ} \mathrm{C}$. This so called infiltration cycle originates from the CFRP production process, where a certain time for resin infiltration of the dry fiber fabric is needed. Afterwards the resin is cured for 2.5 hours at the standard heating temperature of $180^{\circ} \mathrm{C}$ to create a completely cross-linked sample with a curing degree of nearly $100 \%$. To obtain samples with partial curing degrees between $55 \%$ and $80 \%$ the infiltra-
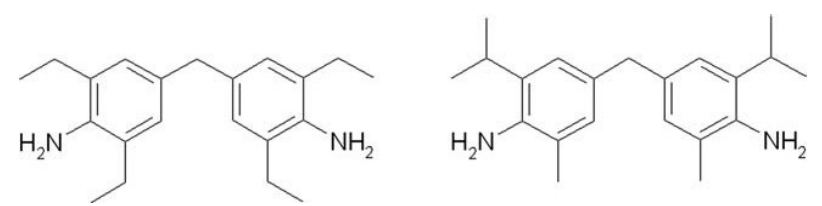

Figure 2. Chemical structure of hardeners 4,4'-methylenebis(2,6-diethylaniline) and 4,4'-methylenebis(2-isopropyl-6-methylaniline).

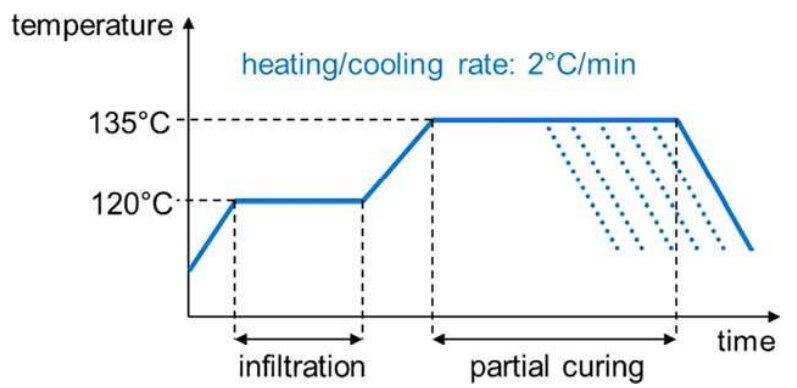

Figure 3. Schematic heating cycle for preparation of partially cross-linked resin samples. [Color figure can be viewed in the online issue, which is available at wileyonlinelibrary.com.]

tion cycle is followed by an isothermal heat treatment at a reduced temperature of $135^{\circ} \mathrm{C}$. The different curing degrees result from different durations of these isothermal heating periods. In Figure 3 the heating cycle for production of partially cross-linked resin samples is shown schematically.

For final curing, the partially cross-linked samples are submitted to a second curing cycle at $180^{\circ} \mathrm{C}$ for 2.5 hours. This second curing step transforms the partially cross-linked resin, e.g. with a curing degree of $60 \%$, to a fully cross-linked resin further nominated as "60/100". The other curing degrees are named equivalently. The complete partial/final heating cycle with combination of partial and final curing is shown schematically in Figure 4 .

Curing Degree, Glass Transition Temperature and Density A 204F1 Phoenix differential scanning calorimeter (Netzsch) was used to measure the heat flow during curing as a function of temperature. To determine the degree of cure of the epoxy resin, the residual heat flow of the cured resin $\Delta H_{R}$ and the total heat flow of the fresh, uncured resin $\Delta H_{T}$ are needed. Both are measured by an analyzing cycle, which comprises a heating ramp from room temperature up to $260^{\circ} \mathrm{C}$ with a heating rate of $5^{\circ} \mathrm{C} / \mathrm{min}$. The heat flow is determined from the resulting DSC curves by integration of the exothermal reaction peak. The curing degree $\alpha$ is determined by

$$
\alpha=1-\frac{\Delta H_{R}}{\Delta H_{T}}
$$

The glass transition temperatures $T_{g}$ of the partially and fully cross-linked epoxy resin samples are determined from the

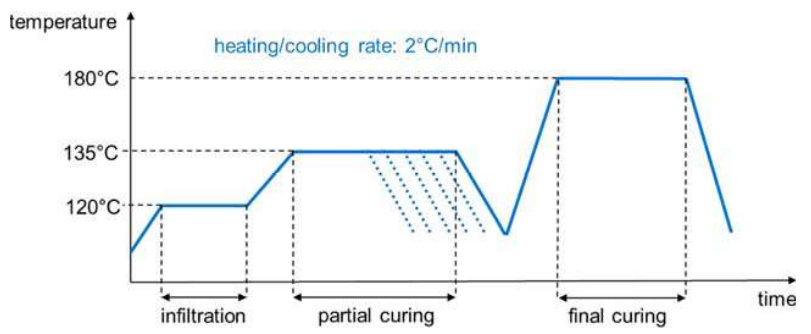

Figure 4. Schematic partial/final heating cycle with combination of partial and final curing for production of finally cross-linked resin samples. [Color figure can be viewed in the online issue, which is available at wileyonlinelibrary.com.] 
modulated DSC curves as the midpoint of the endothermic shift of the reversing heat flow, when the resin passes from the glassy to the rubbery state. The heating rate was $5 \mathrm{~K} / \mathrm{min}$, the modulation amplitude $1 \mathrm{~K}$ and the modulation period $60 \mathrm{sec}$.

Density of the partially and completely cross-linked polymer samples is measured with a helium pycnometer AccuPyc II 1340 (Micromeritics).

For each curing degree at least three resin samples were analyzed.

\section{Micro- and Macromechanical Testing}

Micromechanical elastic moduli of the partially and fully crosslinked epoxy samples were measured by nanoindentation. It allows a laterally resolved modulus analysis with a resolution in the micrometer range. During the loading-unloading indentation cycles the load-displacement curves $F(h)$ are recorded. With the assumption of elastic-plastic material behavior and negligible viscoelastic effects, the micromechanical parameters can be determined from the mainly elastic unloading curve, as described in the following. ${ }^{17-19}$ The influence of viscoelastic material behavior will be discussed afterwards.

The reduced modulus is dependent on the contact area $A$ between indenter and sample and the contact stiffness $S$ and is given by

$$
E_{r}=\frac{\sqrt{\pi}}{2 \beta} \frac{S}{\sqrt{A}}
$$

The contact area $A$ is a function of the contact depth $h_{\mathcal{o}}$ which is defined as

$$
h_{c}=h_{\max }-\varepsilon \frac{F}{S}
$$

$h_{\max }$ is the maximal indenter displacement and $S=d F / d h$ the contact stiffness at the beginning of unloading. $S$ is determined by fitting the unloading curve by the power law $F=\alpha\left(h-h_{p}\right)^{m}$, where $\alpha$ and $m$ are fitting constants and $h_{p}$ is the final depths of the indent after complete unloading. ${ }^{18}$ For the Berkovich indenter tip used the geometry constant $\varepsilon$ is 0.75 and

$$
A \approx 24.5 h_{c}^{2}
$$

The shape correction factor $\beta$ for a Berkovich indenter is unity. ${ }^{18}$ With the knowledge of the Poisson's ratio ration of the sample $v$ and the indenter $v_{i}$ and the modulus of the indenter $E_{i}$, the modulus of the sample $E$ can be determined by

$$
\frac{1}{E_{r}}=\frac{\left(1-v^{2}\right)}{E}+\frac{\left(1-v_{i}^{2}\right)}{E_{i}}
$$

As polymers often are characterized by viscoelastic material behavior, its influence on nanoindentation has to be considered. The delayed viscoelastic material response results in a distortion of the load-displacement curve. ${ }^{17,19,20}$ In particular, because of the gradually increasing deformation after loading and the gradually decreasing deformation after unloading, the unloading curve of a viscoelastic material is more convex than that of elastic materials. This influences the resulting contact stiffness and accordingly the determination of elastic modulus. The influence of viscoelastic effects on the nanoindentation results can be reduced by the insertion of a dwell time between loading and unloading, by slow loading rates and by fast unloading rates. ${ }^{17,19}$ To check the impact of viscoelastic effects on the results presented in this work, nanoindentation measurements were performed with different loading rates between 2 and 10 $\mathrm{mN} / \mathrm{sec}$ and with different dwell times between 0 and $20 \mathrm{sec}$. Low loading rates and a dwell time of more than $2 \mathrm{sec}$ ensure undistorted, reproducible load-displacement curves, and reliable modulus values.

The nanoindentation measurements were performed with a NanoTest 600 nanoindenter (Micromaterials) and an indenter in Berkovich geometry. The load-controlled mode was used with loading and unloading rates of $2 \mathrm{mN} / \mathrm{sec}$. In all measurements a dwell time of $5 \mathrm{sec}$ was inserted between loading and unloading. Before analysis, the raw data of the load-displacement measurement are corrected by the system compliance. To determine the contact stiffness $S$, the unloading curve was fitted with a power law between $0.8 F_{\max }$ and $F_{\max }$. A Poisson's ratio of 0.35 is used. ${ }^{21}$ Average modulus values were obtained by investigating several comparable resin regions. All average values are calculated from more than nine individual values. The measurements were performed on plane surfaces of the polymer samples, which were prepared by a grinding and polishing process. Approximately $50 \mu \mathrm{m}$ of the surface material was ablated by this process.

During indentation compressive loading is applied to the sample surface. Therefore, the measured moduli should be correlated with (macroscopically determined) compressive moduli. For materials with pronounced tensile-compressive anisotropy caution is recommended. For those cases comparing the moduli measured by nanoindentation to macroscopically determined tensile or flexural moduli might be error-prone.

The macroscopic mechanical testing was done with a Kappa 50 DS universal testing machine (Zwick). Tensile-compressive tests in the elastic range were performed on dog bone-shaped resin samples with $1 \mathrm{~mm} / \mathrm{min}$ test speed. Tensile and compressive moduli were determined for at least four specimens of each curing degree. The literature value for the macroscopic tensile modulus of the completely cross-linked resin system is 2.89 $\mathrm{GPa}{ }^{16,21}$ To our knowledge no literature data for the macroscopic compressive modulus exists. For comparable epoxy resin systems different behavior of the compressive moduli is reported. For some resin systems the compressive modulus exceeds the tensile modulus by up to $50 \%,{ }^{22,23}$ for others the compressive and tensile moduli are comparable. ${ }^{24}$

\section{EXPERIMENTAL RESULTS AND DISCUSSION}

In the following the thermo-physical and mechanical investigation of partially and fully cross-linked RTM6 epoxy resin samples is presented. First we describe the dependence of glass transition temperature, density, and micro- and macromechanical moduli on the curing degree. Then the influence of a second, final curing cycle on the resin properties is investigated.

\section{Dependence of Curing Degree on Isothermal Curing} Treatment

By isothermal heating cycles samples with defined partial curing degrees between 55\% and 95\% are produced. Isothermal curing 


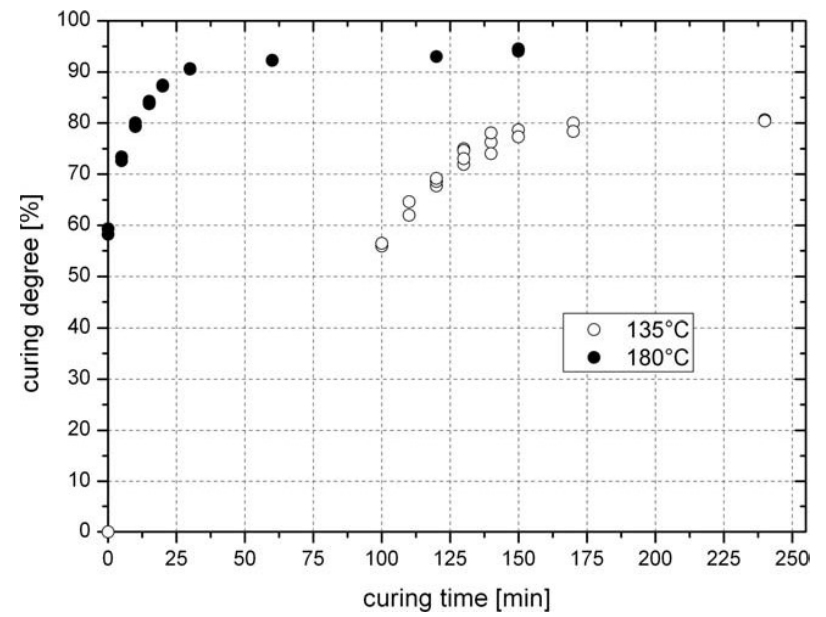

Figure 5. Dependence of curing degree on isothermal heating time for the curing temperatures $135^{\circ} \mathrm{C}$ and $180^{\circ} \mathrm{C}$.

ensures comparable chemical reaction processes for all curing degrees. The curing behavior is determined by an interrelation of physical resin properties, e.g. the viscosity, and chemical reaction kinetics, e.g. the progress of curing. ${ }^{12}$ In Figure 5 the curing degree as a function of the isothermal heating time is displayed for two curing temperatures, namely the reduced heating temperature of $135^{\circ} \mathrm{C}$ and the standard heating temperature of $180^{\circ} \mathrm{C}$.

The curing degree is defined by the duration of isothermal heating. With increasing heating time the curing degree increases monotonically and for long heating periods saturates at a defined maximum curing degree. At the standard heating temperature of $180^{\circ} \mathrm{C}$ curing degrees of maximal $95 \%$ can be reached, which is in agreement with the literature. ${ }^{25} \mathrm{Up}$ to a curing time of 30 minutes $\alpha$ increases rapidly with an average rate of approximately $1.1 \%$ per minute, at longer heating times the curing degree approaches slowly to $95 \%$. Heating at a reduced temperature of $135^{\circ} \mathrm{C}$ allows a maximal curing degree of only $81 \%$. Up to a heating time of 150 minutes $\alpha$ increases monotonically with an average rate of approximately $0.5 \%$ per minute, thus by a factor of two slower than at $180^{\circ} \mathrm{C}$. At higher heating times the curing degree approaches slowly to the maximum value of $81 \%$. The lower reaction rate for the reduced heating temperature of $135^{\circ} \mathrm{C}$ allows a good control of the curing reaction. A defined adjustment of partial curing degrees is possible.

\section{Glass Transition Temperature and its Impact on the Resin's Physical State}

Generally, the glass transition temperature of thermosetting polymers is directly correlated to the curing degree, as a highly cross-linked network needs more energy to allow large-scale molecular motions. In Figure 6 the glass transition temperatures of the partially cross-linked epoxy resin samples cured isothermally at $135^{\circ} \mathrm{C}$ are shown as function of the curing degree.

A nonlinear increase of the glass transition temperature is observed, which can be described by the empirical Di Benedetto equation. The fitting parameter $\lambda$ is 0.4848 , which is in good agreement with values reported for comparable epoxy resin systems by Refs. 26 and 27. The correspondent fit line is included in Figure 6. The glass transition temperature data agree well with the reported measurements on tetra-functional epoxy resin during curing at reduced temperatures. ${ }^{28}$ For a curing degree of $76.5 \% \pm 2.5 \%$ the glass transition temperature crosses the curing temperature, $T_{\mathcal{O}}$ of $135^{\circ} \mathrm{C}$. This has an important impact on the physical properties of the resin and its curing behavior. ${ }^{4,5}$ The gelation point is reached at curing degrees between $40 \%$ and $50 \%$, as can be extrapolated from gel times and curing temperatures given in literature. ${ }^{16}$ Thus all partially cured samples discussed in this work have transformed from liquid to rubber state during curing.

As long as $T_{c}>T_{g}$, namely for samples with $\alpha$ smaller than $76.5 \%$, the samples are still in their rubber state at the end of the isothermal heating procedure. The curing reaction is mainly chemically controlled, in particular for curing degrees below $60 \%{ }^{29}$ When cooling down to room temperature, at $T=T_{g}$ the transition to the glassy state with a freezing of chain motions takes place.

For samples with $\alpha$ higher than 76.5\%, $T_{c}<T_{g}$ applies at the end of isothermal curing and the samples are in their glassy state. Because of this vitrification during cure the reaction now is mostly diffusion controlled and the polymerization rate slows down or essentially stops, limiting the curing degree to $81 \%$ and the glass transition temperature to $165^{\circ} \mathrm{C}$. Additionally, because of annealing at $T_{c}<T_{g}$, physical ageing of the nonequilibrium glassy material with structural relaxation processes starts. The molecular rearrangements result in a gradual decrease of the specific enthalpy of the system, which can be attributed to a decrease of free volume and/or a decrease in molecular conformational energy with time and influence the resin's physical properties. ${ }^{5-7}$ When cooling to room temperature, the sample stays in its glassy state and the structural rearrangements remain.

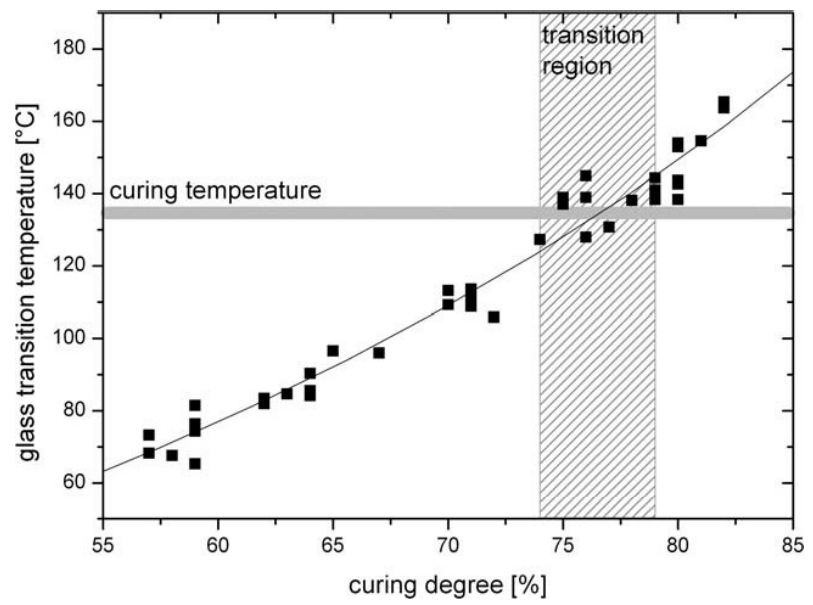

Figure 6. Glass transition temperatures in dependence of curing degree for a heating temperature of $135^{\circ} \mathrm{C}$ together with di Benedetto fit line. The transition region, where the glass transition temperature crosses the heating temperature, is marked. 


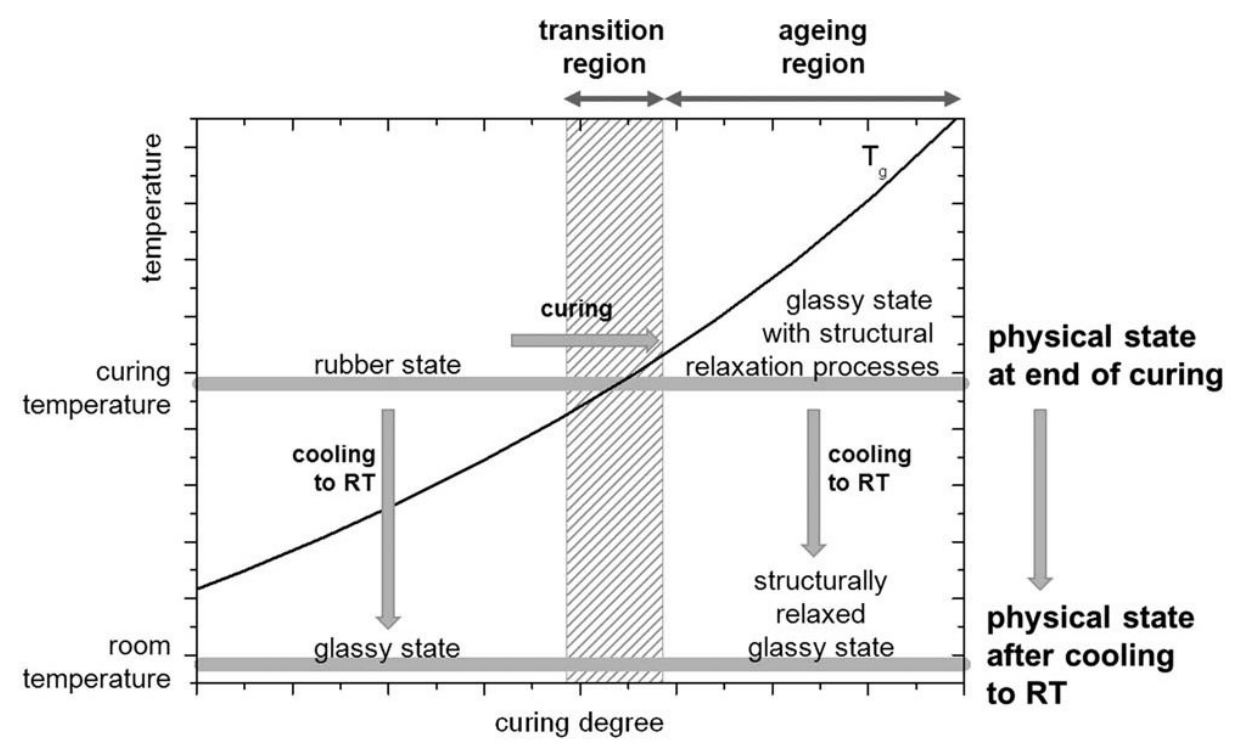

Figure 7. Schematic illustration of different physical states of resin system in dependence of curing degree and temperature.

In the following, for the partially cross-linked samples the region of $76.5 \% \pm 2.5 \%$ curing degree will be called transition region. The region with curing degrees higher than $76.5 \%$ will be nominated as ageing region. In Figure 7 the described physical states of the resin system and the different regions of curing degrees are visualized schematically.

The influence of the different physical states on the properties of the epoxy resin, in particular in the transition and ageing regions, is of high importance for the development of new curing and processing concepts and will be analyzed in the following.

Fully cured samples with a curing degree of about $95 \%$, which are heated at a temperature of $180^{\circ} \mathrm{C}$, reach a glass transition temperature of $209^{\circ} \mathrm{C}$. As $T_{c}<T_{g}$ at the end of cure, these samples are in the glassy state and ageing processes start. Because of the vitrification $\alpha$ is limited to $95 \%$.

\section{Density and Modulus of Partially Cross-Linked Resin}

Density changes during cross-linking of glassy thermosetting network polymers are caused by different, partly opposing effects. On the one hand, the increase of the covalent bond fraction with increasing cross-linking causes volume shrinkage and densification of the polymer. On the other hand the more and more restricted packing of polymer chains because of network formation results in a decrease of density with conversion. $^{10,11}$ Besides, physical ageing during curing at $T_{c}<T_{g}$ causes a volume decrease and thus a densification of the glassy material because of structural relaxation processes. ${ }^{5-7}$ Also an emission of volatile specimen can occur, which causes a mass loss of the sample. ${ }^{9}$ Additionally, also the cooling process from the end-of-curing state (rubbery or glassy) to the room temperature state (glassy) influences the room temperature density of the glassy thermoset. If the cooling process involves a long cooling period within the rubbery state, an efficient chain packing and a high density will result. In contrast, a long cool- ing period within the glassy state allows only minimal molecular rearrangements and thus causes an inefficient chain packing and a low density. ${ }^{11}$ Dependent on the chemical structure of polymer and hardener and the processing conditions, the individual contributions of the described effects determine the final density of the glassy thermoset as a function of the curing degree.

The density is directly correlated to the molecular structure of the polymer, e.g. the crosslinking density and the molecular weight. Therefore it is also closely connected to the modulus of the resin sample, which is determined by the molecular structure, intermolecular forces, intramolecular forces, and relaxation processes. For epoxy systems a similar behavior of modulus and density is reported. ${ }^{9}$

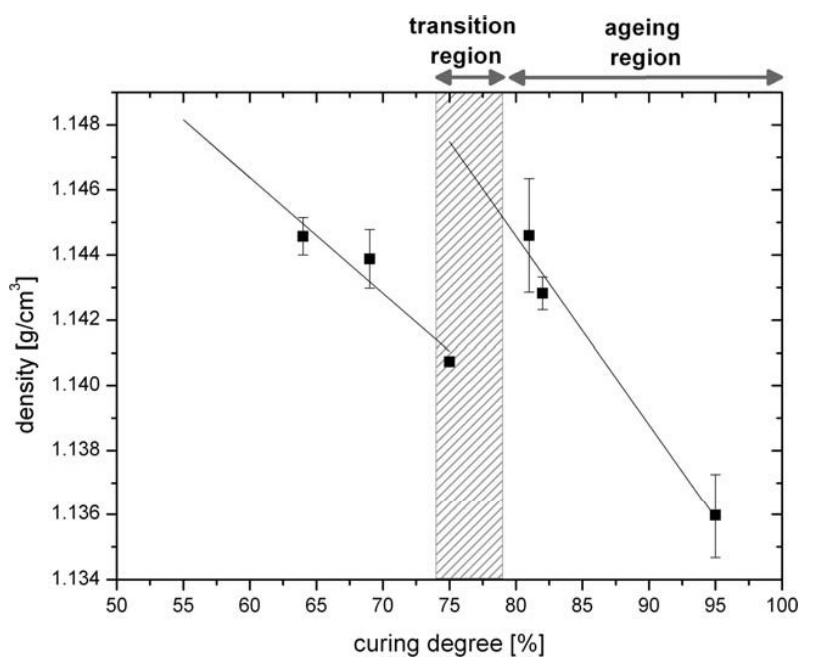

Figure 8. Density of the partially and fully cross-linked polymer samples $\left(T_{c}=135^{\circ} \mathrm{C}\right.$ and $180^{\circ} \mathrm{C}$, respectively) in dependence of the curing degree, together with linear fit lines. 


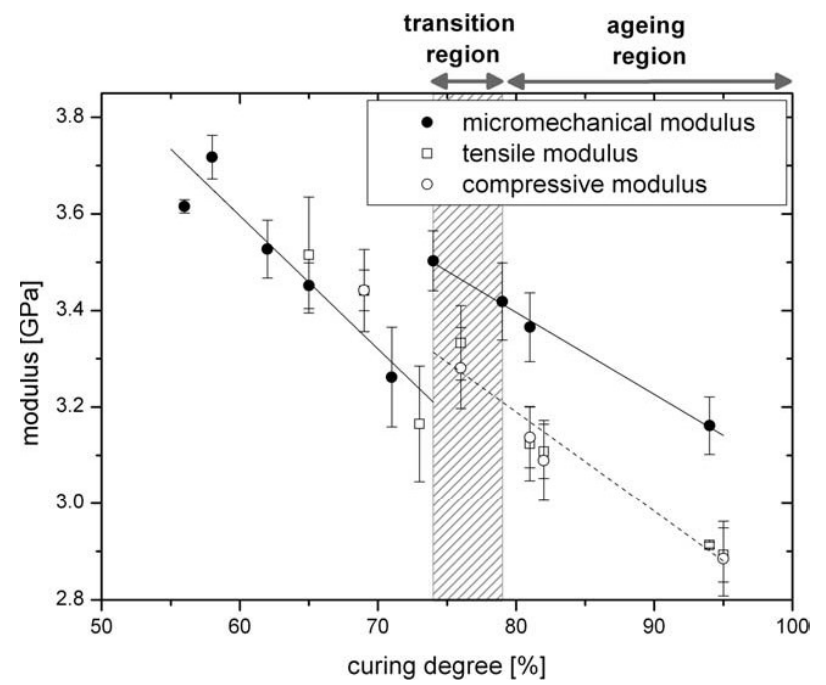

Figure 9. Micro- and macromechanical modulus of partially and fully cross-linked polymer samples $\left(T_{c}=135^{\circ} \mathrm{C}\right.$ and $180^{\circ} \mathrm{C}$, respectively) in dependence of curing degree, together with linear fit lines.

In Figures 8 and 9 the densities and moduli, respectively, of partially and fully cross-linked epoxy resin samples are shown as a function of the curing degree.

To determine the moduli of the epoxy resin samples micromechanical testing by nanoindentation and macromechanical tensile and compressive testing was performed. The high lateral resolution of nanoindentation can be important for interface investigations, e.g. of joined partially cross-linked resins samples, and therefore, represents a promising micromechanical method. A direct comparison of all mechanical results is performed, to evaluate the reliability of micromechanical testing for polymer samples.

For curing degrees below $76.5 \%$ a linear decrease of density and micro- and macromechanical moduli with curing degree is observed, as visualized by the linear fit lines in Figures 8 and 9 . For low curing degrees a rubbery, three-dimensional network with relatively low crosslinking density and low glass transition temperature develops. During cooling to room temperature, rapid molecular rearrangements of not cross-linked molecules occur as long as $T>T_{g}$, and result in relatively low specific volumes and high density and modulus values. With increasing curing degree an increase of crosslinking and glass transition temperature takes place. The growing three-dimensional network and the increase of $T_{g}$ reduces the possibility of molecular arrangements in the rubber state during cooling, increases the specific volume and thus decreases the density and modulus, in agreement with literature. ${ }^{8,10-12,14,15}$ The described effects dominate over the opposing trend of volume shrinkage.

In the transition region an irregularity with a step to higher density and modulus values is found. Here the transition to the glassy state takes place during the curing process once $T_{c}$ crosses $T_{g}$. After the crossing, $T_{c}<T_{g}$ applies and annealing processes start. They allow structural relaxation processes and molecular rearrangements and cause the observed step-like increase of density and modulus. ${ }^{5-7,10}$
For curing degrees above the transition region again a linear decrease of density and modulus is observed with increasing curing degree. Here different, partly opposing effects influence the values. Curing in the glassy state induces further slowly proceeding three-dimensional cross-linking. This is the dominating effect and causes a decrease of density and modulus. Smaller effects are produced by volume shrinkage and structural relaxation processes because of physical ageing, which result in a densification of the material and an increase of modulus. ${ }^{5,7,9}$

Considering the absolute density values, the literature value for a fully cured RTM6 sample is $1.14 \mathrm{~g} / \mathrm{cm}^{3}{ }^{3}{ }^{16}$ This is slightly higher than the measured value of $1.1360 \pm 0.00129 \mathrm{~g} / \mathrm{cm}^{3}$, possibly because of minor differences in the curing cycle.

Overall a density decrease of $1.01 \%$ is observed with increasing curing degree in the range between $55 \%$ and $95 \%$ curing degree. The result is in agreement with literature values, where for other three-dimensional epoxy resin systems also a decrease of density with increasing curing degree is described for a comparable range of curing degrees in the glassy state. The density decreases reported are between $0.10 \%$ and $0.41 \%$ and are smaller than our value, but of the same order of magnitude. ${ }^{9-12}$ An irregularity in the density values is described in Ref. 6, however, at much higher curing degrees and within the glassy state.

Focusing on the absolute values of micro- and macromechanical moduli, some points should be noted. First, the agreement of macroscopic tensile and compressive moduli is very good. No tensile-compressive anisotropy is observed for the RTM6 resin system. Consequently, a comparison of moduli from nanoindentation to both, tensile and compressive moduli is possible. Comparison to literature data shows a very good agreement of the macroscopic tensile modulus of the fully cross-linked resin sample ( $95 \%$ curing degree) of $2.893 \pm 0.0563 \mathrm{GPa}$ to the literature value of $2.890 \mathrm{GPa}^{16,21}$

Secondly, macromechanical testing shows that the modulus decreases by $22.9 \%$ with increasing curing degree in the range between 55\% and 95\%. Micromechanical testing exhibits a decrease of modulus of $15.9 \%$ in the same range of curing degrees. These results are in qualitative agreement with literature, where also a decrease of modulus with curing degree is described..$^{8,11,14,15}$ The reported values lie between $9.3 \%$ and $41.8 \%$.

Finally, the agreement of the absolute values of micro- and macromechanical moduli differs for the two regimes of curing degrees. For curing degrees below the transition region, the absolute modulus values of micro- and macromechanical testing are in very good agreement. In contrast, the step height in the transition region and the modulus values in the ageing regime differ clearly. The macromechanical moduli show an absolute step height of $0.10 \mathrm{GPa}$, which is by a factor of three smaller than that of the micromechanical moduli of $0.29 \mathrm{GPa}$. Consequently, for high curing degrees the absolute micromechanical moduli are clearly higher than the macromechanical moduli. They differ by about $0.25 \mathrm{GPa}$. One possible reason for the differences between micro- and macromechanical moduli in the ageing regime above the transition region could be an enhanced 


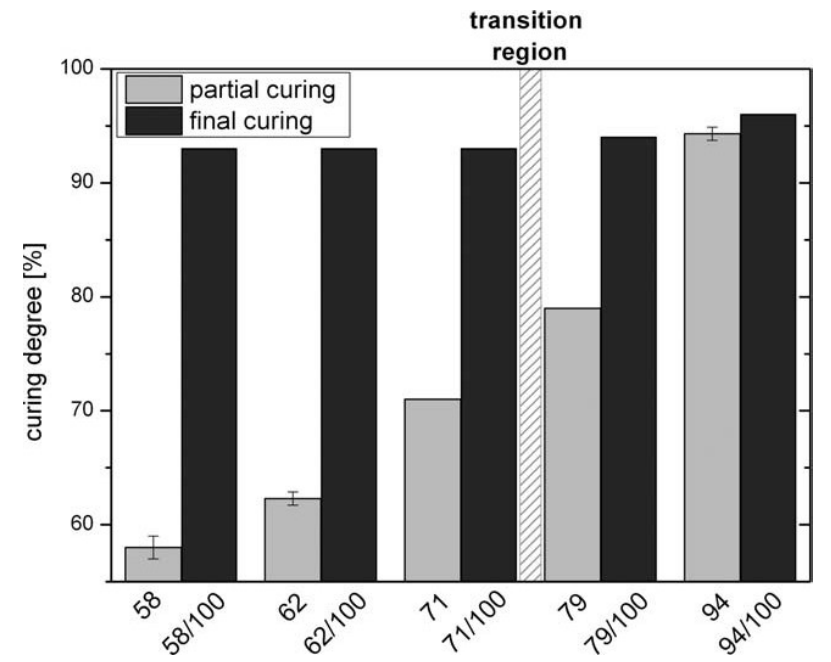

Figure 10. Curing degrees of partially and fully cured resin samples and corresponding partially/finally and fully/finally cured samples.

surface ageing of the polymers, as described in literature. ${ }^{30,31}$ However, this description, which would result in an increased surface modulus and surface density, cannot explain our experimental results as will be explained in the following. As nanoindentation probes the surface properties, the micromechanical moduli would be affected by such surface degradation. In contrast, bulk properties as macromechanical modulus and density should only be marginally influenced by an aged surface layer and should show a smaller step in the transition region and smaller values in the ageing regime. However, a similar relative step height (defined as ratio between the step height in the transition region and the total decrease between $55 \%$ and $76.5 \%$ curing degree) of micromechanical modulus (55.1\%) and density $(50.0 \%)$ is observed. It is clearly higher than that of the macromechanical moduli of $13.5 \%$. Thus, the differences between micro- and macromechanical testing cannot be caused by surface ageing effects. The good agreement between density and micromechanical modulus suggests that micromechanical testing by nanoindentation is more sensitive to the molecular arrangement of the polymer, in particular to the molecular relaxations because of annealing, than macromechanical testing. The mechanical testing conditions seem to allow an emphasis of different modulus contributions, i.e. the molecular structure or molecular forces.

Nanoindentation of polymer surfaces thus represents a very valuable tool for modulus and density investigation of polymers with high lateral resolution that expands the possibilities of macroscopic mechanical testing.

The irregularity of density and modulus observed in the transition region is a particular feature of our partially cross-linked samples, which to our knowledge is not described in literature so far. It is caused by the specific isothermal curing cycles, which allow a transition from rubber to glassy state during curing. This transition influences the properties of the partially and fully cross-linked thermosetting samples even in the glassy state after cooling down to room temperature and represents a promising effect for defined adjustment of physical resin prop- erties. The relatively high moduli above the step within the transition region are typical for much lower cross-linking degrees and could be advantageous during further curing treatments or processing.

\section{Investigation of Partially/Finally Cured Resin Samples}

All partially cross-linked resin samples are submitted to a second, final curing cycle at $180^{\circ} \mathrm{C}$. In the CFRP production process this final heating cycle is responsible for the complete curing of partially cross-linked resin samples. The physical properties of the partially/finally cured samples are analyzed, to check the influence of the partial curing cycle on the final resin properties. In Figures 10 and 11 the curing degrees and the glass transition temperatures of the partially/finally cured samples (called 58/100,62/100,71/100, and 79/100) and the fully/finally cured sample (called 94/100) in comparison to that of the corresponding partially (called 58, 62, 71, and 79) and fully (94) cured resin samples are shown, respectively.

All partially/finally cured resin samples achieve very similar curing degrees between $93 \%$ and $94 \%$ and very similar glass transition temperatures between $209.2^{\circ} \mathrm{C}$ and $212.4^{\circ} \mathrm{C}$. As the glass transition temperature of all partially cured samples lies below $180^{\circ} \mathrm{C}$, these samples transform to rubber state during final curing. This allows further cross-linking and causes the observed strong increases of curing degrees and glass transition temperatures. All partially/finally cured resin samples thus seem to be equivalent in terms of curing degree and glass transition temperature, independent from their partial curing degree. Besides, they exhibit values similar to that of the fully cross-linked 94sample $\left(\alpha=94.3 \%, T_{g}=209.0^{\circ} \mathrm{C}\right)$. This is an important point for application of partially/finally cured epoxy resins, as it guarantees conformance of thermo-physical properties of partially/ finally cured samples with conventionally fully cured resin samples.

The fully cross-linked 94-sample has a glass transition temperature above the final curing temperature of $180^{\circ} \mathrm{C}$. Therefore this

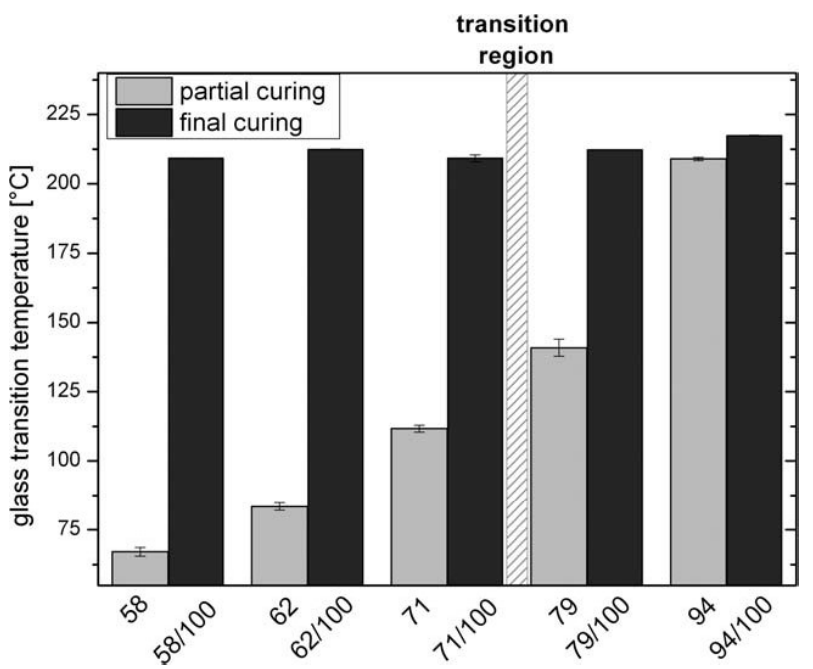

Figure 11. Glass transition temperatures of partially and fully cured resin samples and corresponding partially/finally and fully/finally cured samples. 


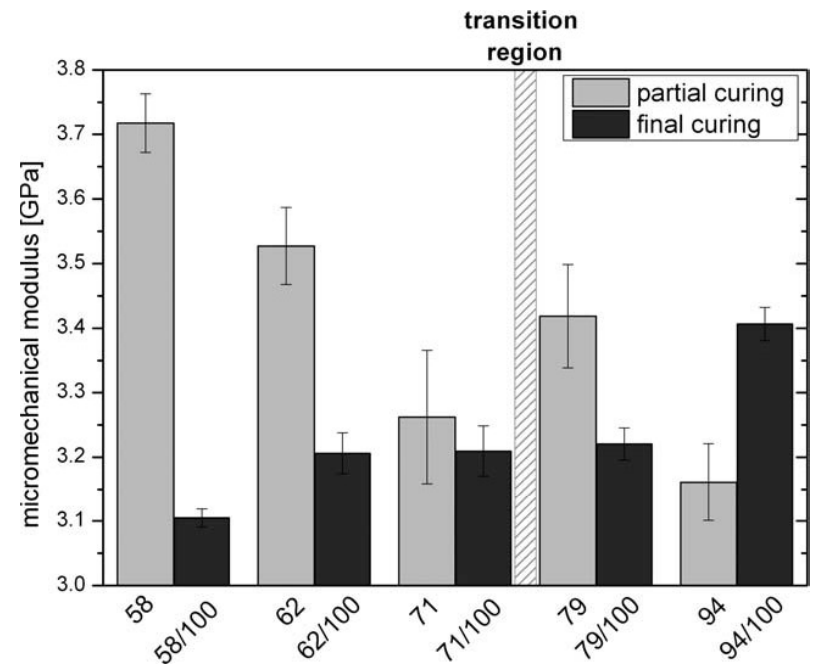

Figure 12. Micromechanical moduli of partially and fully cured resin samples and corresponding partially/finally and fully/finally cured resin samples.

sample stays in its glassy state during final curing and only little increase of curing degree and glass transition temperature is possible. The resulting 94/100-sample exhibits a curing degree of $96 \%$ and a glass transition temperature of $217^{\circ} \mathrm{C}$, which are slightly higher than the values obtained by combined partially and finally curing.

Figure 12 shows the micromechanical moduli of the partially/ finally and fully/finally cured resin samples, in comparison with the corresponding values of the partially or fully cured samples.

For all partially cured resin samples a decrease of modulus with final curing treatment at $180^{\circ} \mathrm{C}$ is observed. As described, heating at $T_{c}>T_{g}$ results in further cross-linking and the correspondent reduction of modulus. ${ }^{8}$ The strongest effect is detected for the 58/100-sample. With its low partial curing degree it exhibits the strongest increase in curing degree and thus the strongest built up of three-dimensional network. The smallest change of modulus is observed for the 71/100-sample, which has a relatively small modulus already in its partially cured state. The micromechanical moduli of all partially/finally cured samples $(58 / 100,62 / 100,71 / 100,79 / 100)$ range between 3.11 and $3.22 \mathrm{GPa}$, and are very similar to that of the fully cured 94-sample of $3.16 \mathrm{GPa}$. The combination of partial and final curing cycle thus creates elastic properties of the epoxy resin that are comparable to that after conventional full curing, which is in agreement with literature data. ${ }^{11}$ This is of high relevance for application of partially cross-linked epoxy resins, as the combined curing treatment guarantees mechanical properties similar to that of a conventional single curing step. Only small mechanical mismatch is expected for samples with different heating histories, e.g. for joining of partially/finally and fully cured (94) resin regions.

The step-like increase of modulus, observed for the partially cross-linked samples, does not exist in the partially/finally cured state. No irregularity of modulus is observed in the transition region. The second heat treatment removes the distinctions cre- ated by the different ageing conditions of the partial curing cycles. All finally cured samples are in their glassy state at the end of final curing and thus are aged by similar heat treatments.

For the fully cross-linked sample (94) an increase of modulus is observed after the final heat treatment (94/100). The modulus increases from 3.16 to $3.41 \mathrm{GPa}$. Because of the high glass transition temperature of the 94 -sample of $209.0^{\circ} \mathrm{C}$, final curing takes place at $T_{c}<T_{g}$. This results in an annealing process with structural relaxations and the corresponding increase in modulus of the 94/100-sample. A not negligible mechanical mismatch of moduli of $0.25 \mathrm{GPa}$ between the 94 and $94 / 100$ samples is observed. A combination of fresh resin with completely cured resin and following final curing, e.g. for joining, thus is not favorable. Also CFRP processing steps, which involve a thermal impact on a conventional fully cross-linked resin sample, e.g. the application of functional layers, will cause a change of mechanical properties in the heated resin regions. The resulting embrittlement of the surface regions can weaken the CFRP component.

\section{SUMMARY}

Partial cross-linking of tetra-functional thermosetting RTM6 epoxy resin retains particular chemical reactivity of the polymer and marks a promising way for new curing and processing routes for CFRP production, e.g. the development of energy efficient curing cycles, joining of CFRP components or application of functional layers. A second final curing step guarantees the complete cross-linking of the resin.

Knowledge of the interrelation between basic thermo-physical and mechanical properties of the partially and finally crosslinked resins is necessary to develop specifically adapted curing cycles for different applications. With this objective the investigation of curing degree, glass transition temperature, density, and micro- and macromechanical modulus of partially and finally cured RTM6 epoxy resin samples in the glassy state was performed. The results show a significant dependence of the physical properties on the curing history and the curing degree. Density and modulus show on the one hand a decrease with increasing curing degree that is caused by the cross-linking reaction of epoxy resin and hardener and the built-up of a threedimensional network. On the other hand an irregularity in the so called transition region between $74 \%$ and $79 \%$ curing degree is observed, which can be correlated to the transition from rubber to glassy state during the thermal treatment. The resulting ageing in the glassy state is connected to structural relaxation processes, which enhance the density and modulus of the polymer. The step-like irregularity of physical properties is a typical feature of samples manufactured with our isothermal, low temperature curing cycle and demonstrates the options of adapted curing treatments for defined adjustment of physical properties. The relatively high modulus and density above the step is typical for resins with much lower curing degrees and offers new possibilities, e.g. for joining of partially cross-linked and fresh resin parts. After final curing of the partially cross-linked resins, the resulting curing degree, glass transition temperature and 
modulus are similar to that of a conventional fully cured sample and only negligible mismatch of the thermo-physical and mechanical properties exists.

The investigations demonstrate the impact of the thermal history on the basic physical properties of tetra-functional epoxy resins systems and the wide field of potential applications of partially cross-linked epoxy resin systems.

\section{ACKNOWLEDGMENTS}

Thanks to Günter Obermeier and Andreas Monden for valuable scientific contributions.

\section{REFERENCES}

1. May, C. A. Epoxy Resins: Chemistry and Technology, 2nd ed.; M. Dekker: New York, 1988.

2. Mohan, P. Polym. Plast. Technol. Eng. 2013, 52, 107.

3. Sause, M.; Llopard Prieto, L.; Scholler, J.; Horn, S.; Moosburger-Will, J.; Horny, R. Patent DE 102011010384 A1.

4. Yoon, S. S.; Yu, W. J.; Kim, H. C. J. Mater. Sci. Lett. 1992, 11, 1392.

5. Montserrat, S. J. Appl. Polym. Sci. 1992, 44, 545.

6. Wang, X.; Gillham, J. K. J. Appl. Polym. Sci. 1993, 47, 447.

7. Odegard, G. M.; Bandyopadhyay, A. J. Polym. Sci. Part B: Polym. Phys. 2011, 49, 1695

8. Ellis, B.; Found, M. S.; Bell, J. R. J. Appl. Polym. Sci. 2001, $82,1265$.

9. Venditti, R. A.; Gillham, J. K. J. Appl. Polym. Sci. 1995, 56, 1687.

10. Wang, X.; Foltz, V. J. Macromolecules. 2001, 34, 681.

11. Detwiller, A. T.; Lesser, A. J. J. Appl. Polym. Sci. 2010, 117, 1021.

12. Enns, J. B.; Gillham, J. K. J. Appl. Polym. Sci. 1983, 28, 2831.
13. Wang, X.; Gillham, J. K. J. Appl. Polym. Sci. 1993, 47, 425.

14. Morel, E.; Bellenger, V.; Bocquet, M.; Verdu, J. J. Mater. Sci. 1989, 24, 69.

15. White, S. R.; Mather, P. T.; Smith, M. J. Polym. Eng. Sci. 2002, 42, 52.

16. Hexcel Company. Product literature to HexFlow RTM6®.

17. Menčík, J.; He, L. H.; Němeček, J. Polym. Test. 2011, Bd. 30, 101.

18. Oliver, W. C.; Pharr, G. M. J. Mater. Res. 2004, 19, 3.

19. Briscoe, B. J.; Fiori, L.; Pelillo, E. J. Phys. D Appl. Phys. 1998, 31, 2395.

20. Cheng, Y.; Cheng, C. Appl. Phys. Lett. 2005, 87, 111914.

21. Brauner, C.; Block, T. B.; Purol, H.; Herrmann, A. S. J. Compos. Mater. 2012, 46, 993.

22. Behzadi, S.; Jones, F. R. J. Macromol. Sci. Part B: Phys. 2005, 44, 993.

23. Ciba Speciality Chemicals. Product literature to Araldite MY 721.

24. Kozey, V. V.; Kumar, S. J. Mater. Res. 1994, 9, 2717.

25. Navabpour, P.; Nesbitt, A.; Degamber, B.; Fernando, G.; Mann, T.; Day, R. J. Appl. Polym. Sci. 2006, 99, 1658.

26. Karkanas, P. I.; Partridge, I. K. J. Appl. Polym. Sci. 2000, 77, 2178.

27. Sadeghinia, M.; Jansen, K. M. B.; Ernst, L. J. Int. J. Adhes. Adhes. 2012, 32, 82.

28. Cizmecioglu, M.; Gupta, A.; Fedors, R. F. J. Appl. Polym. Sci. 1986, 32, 6177.

29. Karkanas, P. I.; Partridge, I. K. J. Appl. Polym. Sci. 2000, 77, 1419.

30. Monney, L., Bole, J.; Dubois, C.; Chambaudet, A. Polym. Degrad. Stab. 1999, 66, 17.

31. Moosburger-Will, J.; Jäger, J.; Horn, S.; Wellhausen, C. Polym. Test. 2012, 31, 1008. 